\title{
In human gestational diabetes mellitus congenital malformations are related to pre-pregnancy body mass index and to severity of diabetes
}

\author{
A. García-Patterson ${ }^{1}$ L. Erdozain ${ }^{1}$ - G. Ginovart $^{2}$ • J. M. Adelantado ${ }^{3}$ J. M. Cubero ${ }^{1}$ G. Gallo ${ }^{1}$ \\ A. de Leiva ${ }^{1,4} \cdot \mathbf{R}$. Corcoy ${ }^{1,4}$ \\ ${ }^{1}$ Department of Endocrinology and Nutrition, Hospital of the Holy Cross and St. Paul, Autonomous University of Barcelona, \\ Barcelona, Spain \\ ${ }^{2}$ Department of Pediatrics, Hospital of the Holy Cross and St. Paul, Autonomous University of Barcelona, Spain \\ ${ }^{3}$ Department of Gynecology and Obstetrics, Hospital of the Holy Cross and St. Paul, Autonomous University of Barcelona, Spain \\ ${ }^{4}$ Centre Network of the Carlos III Health Institute, Madrid, Spain
}

\section{Abstract}

Aims/hypothesis. This study analysed the relationship between congenital malformations (CM) and severity of gestational diabetes mellitus.

Methods. A cohort of 2060 infants of mothers with gestational diabetes was studied. Universal screening and $3^{\text {rd }}$ Workshop-Conference criteria were used to diagnose gestational diabetes. The severity of diabetes was assessed on the basis of previous hyperglycaemia, blood glucose values in diagnostic OGTT, area under the glucose curve, gestational age and $\mathrm{HbA}_{1} \mathrm{c}$ at diagnosis, insulin requirements during pregnancy, and OGTT after delivery. Potentially confounding variables (age, pre-pregnancy BMI, smoking) were considered. The relationship of potential predictors with $\mathrm{CM}$ was analysed with several multivariate logistic regression analyses.

Results. The rate of CM was $6 \%$ for minor and $3.8 \%$ for major malformations $(1.4 \%$ heart, $0.8 \%$ renal/urinary, $0.7 \%$ skeletal, $0.3 \%$ hypospadias, $0.2 \%$ central nervous system, $0.2 \%$ cleft lip/palate, $0.1 \%$ digestive tract, $0.3 \%$ other). In the final models, forward logistic regression analysis identified pre-pregnancy BMI as the predictor of CM (area under receiver operating characteristic curve 0.616); in the backward analysis additional predictors were 1-h blood glucose in diagnostic OGTT and gestational age at diagnosis (area under receiver operating characteristic curve 0.646). Both BMI and severity of gestational diabetes were predictors of heart and minor CM, whereas BMI predicted renal/urinary $\mathrm{CM}$ and severity of diabetes predicted skeletal CM.

Conclusions/interpretation. In these infants of mothers with gestational diabetes, severity of diabetes and pre-pregnancy BMI were predictors of $\mathrm{CM}$, in accordance with the well-documented pathogenic role of BMI (in the general population) and hyperglycaemia (in diabetic pregnancy). BMI was the main predictor of more prevalent CM. [Diabetologia (2004) 47:509514]

Keywords Gestational diabetes mellitus - Congenital malformations $\cdot$ Body mass index $\cdot$ Hyperglycaemia
Received: 18 March 2003 / Revised: 18 November 2003

Published online: 10 February 2004

(C) Springer-Verlag 2004

R. Corcoy ( $)$

Department of Endocrinology and Nutrition,

Hospital of the Holy Cross and St. Paul,

Autonomous University of Barcelona,

Avinguda Sant Antoni $\mathrm{M}^{\mathrm{a}}$ Claret, 167,

Barcelona 08025, Spain

E-mail: rcorcoy@hsp.santpau.es

Abbreviations: CM, congenital malformations . GDM, gestational diabetes mellitus ·

ROC, receiver operating characteristic
Pre-pregnancy diabetes mellitus is a well-known risk factor for congenital malformations (CM) $[1,2]$ related to hyperglycaemia during the first weeks of pregnancy when embryogenesis takes place [3]. This higher rate of $\mathrm{CM}$ is reduced in women receiving pre-pregnancy care [4]. An increased rate of CM has also been reported in women with gestational diabetes mellitus (GDM) [5] and related with fasting blood glucose in three studies [6, 7, 8]. Results were adjusted for maternal age [6] or parity, prior GDM and several glycaemic parameters $[7,8]$. The aim of this study was to analyse whether there is a relationship between CM and severity of GDM in a different population. 


\section{Subjects and methods}

Study cohort and diagnostic methods. The study cohort comprised consecutive infants of mothers with a documented diagnosis of GDM, born between January 1986 and July 2002 at 22 complete weeks of gestation or later at a single institution (Hospital of the Holy Cross and St Paul, Barcelona, Spain). The diagnosis of GDM was in accordance with the $3^{\text {rd }}$ Workshop-Conference on GDM criteria [9]. Maternal ethnicity was: White $98.7 \%$, Black 0.4\%, Arab 0.2\%, Asian 0.2\%, Hispanic $0.2 \%$, Gipsy $0.2 \%$, not available $0.1 \%$. Severity of GDM was assessed on the basis of: (i) previous hyperglycaemia [GDM, IGT, non-diagnostic hyperglycaemia (fasting plasma glucose $>6.1 \mathrm{mmol} / \mathrm{l},<7.8 \mathrm{mmol} / \mathrm{l}$ according to the World Health Organisation (1985) criteria [10] that were applicable at the time the patients were attended)]; (ii) blood glucose in the diagnostic OGTT (fasting and 1-, 2-, and 3-h postload blood glucose, area under the curve of glucose, number of abnormal values); (iii) gestational age at diagnosis; (iv) $\mathrm{HbA}_{1} \mathrm{c}$ at diagnosis; (v) insulin requirements during pregnancy; and (vi) OGTT after delivery according to WHO criteria [11]. Maternal age [12], prepregnancy BMI [13] and smoking habits [14] in the first trimester were considered potentially confounding variables. No systematic information was recorded on alcohol consumption and vitamin supplementation, so these variables were not included in the model. This study was carried out in accordance with the Declaration of Helsinki as revised in 2000. Institutional Review Board approvement was not required for observational studies at the time this study was done.

Newborn infants were assessed by a neonatologist before hospital discharge, and image studies were scheduled within the first weeks of life if a CM was suspected. Major CM were defined as those which were life-limiting, i.e., caused significant functional or cosmetic impairment or required surgery. Minor CM were defined as anomalies without serious medical or cosmetic significance and occurring in less than $4 \%$ of the background population.

Statistical analysis. SPSS 10.0 for Windows (SPSS Inc., Chicago, Ill., USA) was used for analysis. To explore the relationship between CM and quantitative variables, the latter were transformed into tertiles. The relationship of each potentially predictive variable with $\mathrm{CM}$ was analysed using a chi square test and predictive variable categories with a similar CM rate were grouped (Table 1). Finally, several multivariate logistic regression models were constructed with $\mathrm{CM}$ as the dependent variable, using both forward and backward methods. In the first analysis, all predictive variables were included (previous hyperglycaemia, fasting, 1-, 2-, and 3-h blood glucose, number of abnormal values, area under the OGTT curve and gestational age at diagnosis, $\mathrm{HbA}_{1} \mathrm{c}$ at diagnosis, insulin requirements during pregnancy, postpartum OGTT, pre-pregnancy BMI, maternal age and smoking habits). The number of cases was limited to 983 . As the number of cases included was less than $50 \%$ of the original population, additional analyses were done in this subgroup, after excluding variables with missing cases (postpartum OGTT, smoking habits and $\mathrm{HbA}_{1} \mathrm{c}$ at diagnosis). As the predictors of $\mathrm{CM}$ did not change in the various models, additional analyses were done excluding postpartum OGTT, smoking habits and $\mathrm{HbA}_{1} \mathrm{c}$ as predictive variables $(n=1938$, $94 \%$ of the total cohort). This strategy was used to explore the predictors of major CM (one or more). For the prediction of minor CM (one or more) and individual major CM, forward and backwards multiple logistic regression analyses were directly carried out excluding variables with missing cases (postpartum OGTT, smoking habits and $\mathrm{HbA}_{1} \mathrm{c}$ ). Models offering the highest prediction [area under the receiver operating char- acteristic (ROC) curve] were retained as final. Significance was set at a $p$ value less than 0.05 .

\section{Results}

The rate of CM in this cohort of 2060 infants (1894 singletons, 142 twins, 24 triplets) born to mothers with GDM was: minor malformations (one or more) $6 \%$, and major malformations (one or more) $3.8 \%$ (1.4\% heart, $0.8 \%$ renal/urinary, $0.7 \%$ skeletal, $0.3 \%$ hypospadias, $0.2 \%$ central nervous system, $0.2 \%$ cleft lip/palate, $0.1 \%$ digestive tract, $0.3 \%$ other).

In the bivariate analysis there was a trend towards a higher rate of major CM with increasing fasting blood glucose and a positive relationship with previous hyperglycaemia, pre-pregnancy BMI, lower gestational age at diagnosis, number of abnormal values in the diagnostic OGTT, higher $\mathrm{HbA}_{1} \mathrm{c}$ at diagnosis of GDM, and postpartum OGTT (Table 1). In the logistic regression analyses including all potentially predictive variables the identified predictor was four abnormal values in the diagnostic OGTT and/or overt diabetes during pregnancy versus two to three abnormal values in the diagnostic OGTT (odds ratio 4.93, CI 95\%: 1.61-15.05). The same predictor was identified with forward and backward analysis and after sequentially excluding postpartum OGTT, smoking habits and $\mathrm{HbA}_{1} \mathrm{c}$ in this group (Table 2). With a forward analysis done excluding variables with missing values in the whole cohort, pre-pregnancy BMI was the single predictor of major $\mathrm{CM}$ with a positive association, whereas in the backward analysis, 1-h blood glucose in the diagnostic OGTT (positive association, non-significant for individual tertiles) and gestational age at diagnosis (non-significant negative association) were also included in the model (Table 3). As the area under the ROC curve was somewhat higher, this last model was retained as final (Table 4).

Forward and backward analyses identified the same predictors of major $\mathrm{CM}$ affecting the heart: pre-pregnancy BMI (positive association, significant for the $2^{\text {nd }}$ tertile, non-significant for the $3^{\text {rd }}$ ), 2-h blood glucose in the diagnostic OGTT (negative association, significant for the $2^{\text {nd }}$ tertile, non-significant for the $3^{\text {rd }}$, four abnormal values in the diagnostic OGTT and/or overt diabetes during pregnancy (positive association), and gestational age at diagnosis (negative association).

For renal/urinary major CM, pre-pregnancy BMI was the only predictor (positive association, significant for the $3^{\text {rd }}$ tertile, non-significant the $2^{\text {nd }}$ ) in forward logistic regression analysis, whereas maternal age was also a predictor (non-significant positive association) in backward analysis. This model was retained as final, as the ROC area was higher.

As to skeletal major CM, no predictors were identified in forward analysis whereas fasting blood glucose 
Table 1. Congenital malformations in infants of women with gestational diabetes mellitus. Relationship with potential predictors (bivariate analysis)



a The significance provided refers to the comparison within all categories of a variable

b Blood glucose values in diagnostic OGTT c SD around the mean of control non-pregnant population $\mathrm{CM}$, congenital malformations; $\mathrm{BG}$, blood glucose values; DM, diabetes mellitus; NA, not applicable displayed a positive association (non-significant for individual tertiles) in backward analysis. As the area under the ROC curve was significant, this model was retained.

No predictors were identified for hypospadias, cleft lip/palate and major $\mathrm{CM}$ of the central nervous system, digestive tract and others.

In forward analysis, no predictors were identified for minor CM, whereas backward analysis identified pre-pregnancy BMI (positive association, non-significant for individual tertiles), 2-h blood glucose in the diagnostic OGGT (positive association, non-significant for individual tertiles) and 3-h blood glucose in the diagnostic OGTT (negative association). As the area under the ROC curve was significant, this model was retained. 
Table 2. Multivariate logistic regression analysis to predict congenital malformations in the subset of women with gestational diabetes and all predictor variables

\begin{tabular}{|c|c|c|c|c|}
\hline & \multicolumn{4}{|c|}{ Potentially predictive variables fed to the model } \\
\hline & All variables & $\begin{array}{l}\text { All variables, } \\
\text { excluding postpartum } \\
\text { OGTT }\end{array}$ & $\begin{array}{l}\text { All variables, excluding } \\
\text { postpartum OGTT } \\
\text { and smoking }\end{array}$ & $\begin{array}{l}\text { All variables, excluding } \\
\text { postpartum OGTT, } \\
\text { smoking and } \mathrm{HbA}_{1 \mathrm{c}}\end{array}$ \\
\hline Number of cases & 983 & 983 & 983 & 983 \\
\hline $\begin{array}{l}n \text { of abnormal values } \\
\text { in the diagnostic OGTT }\end{array}$ & $95 \%$ CI (1.61-15.05) & $95 \%$ CI (1.61-15.05) & $95 \%$ CI (1.61-15.05) & $95 \%$ CI (1.61-15.05) \\
\hline ROC curve area & 0.546 & 0.546 & 0.546 & 0.546 \\
\hline \multicolumn{5}{|l|}{ Backward analysis } \\
\hline $\begin{array}{l}\text { Independent predictor: } \\
n \text { of abnormal values } \\
\text { in the diagnostic OGTT }\end{array}$ & $\begin{array}{l}\mathrm{OR}=4.928 \\
95 \% \mathrm{CI}(1.61-15.05)\end{array}$ & $\begin{array}{l}\mathrm{OR}=4.928 \\
95 \% \text { CI }(1.61-15.05)\end{array}$ & $\begin{array}{l}\mathrm{OR}=4.928 \\
95 \% \text { CI }(1.61-15.05)\end{array}$ & $\begin{array}{l}\mathrm{OR}=4.928 \\
95 \% \text { CI }(1.61-15.05)\end{array}$ \\
\hline
\end{tabular}

The table shows models resulting after exclusion of variables with missing cases in the whole group. OR, odds ratio; ROC, receiver operating characteristic

Table 3. Multiple logistic regression analysis to predict congenital malformations in offspring of women with gestational diabetes mellitus

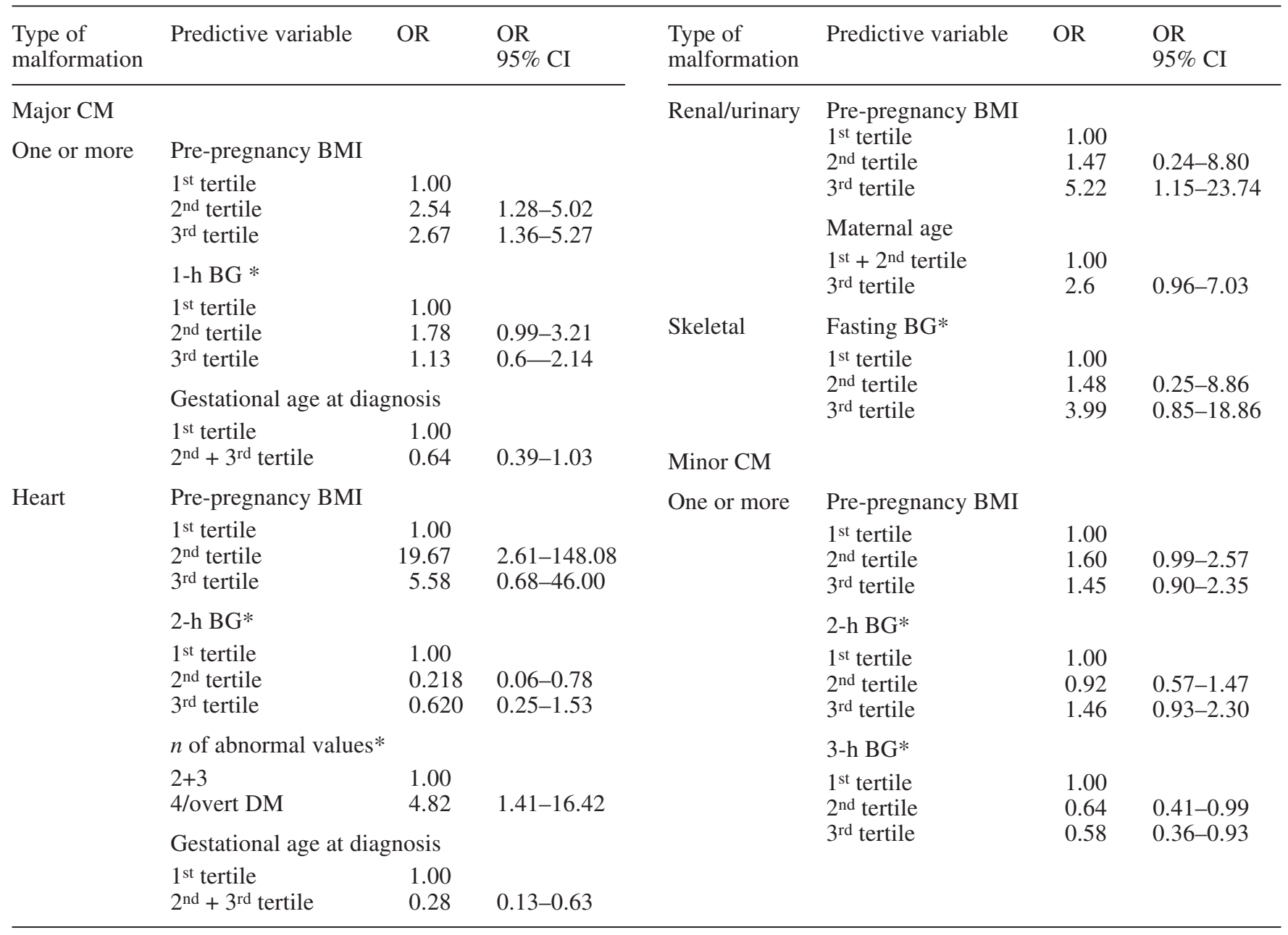

All potentially predictive variables were fed (backwards analysis) to the model with the exception of those with missing values (postpartum OGTT, smoking habit and $\left.\mathrm{HbA}_{1} \mathrm{c}\right)(n=1938)$.
* BG, blood glucose at diagnostic OGTT. CM, congenital malformation; OR, odds ratio; ROC, receiver operating characteristic; DM, diabetes mellitus 
Table 4. Multiple logistic regression analysis: overall prediction of models described in Table 3

\begin{tabular}{lcll}
\hline $\begin{array}{l}\text { Type of } \\
\text { malformation }\end{array}$ & $\begin{array}{l}p \text { of the } \\
\text { model }\end{array}$ & ROC area & $\begin{array}{l}\text { ROC area } \\
95 \% \text { CI }\end{array}$ \\
\hline Major CM & & & \\
One or more & $<0.01$ & 0.646 & 0.586 to 0.706 \\
Heart & 0.01 & 0.814 & 0.747 to 0.881 \\
Renal/urinary & 0.01 & 0.751 & 0.663 to 0.840 \\
Skeletal & 0.01 & 0.656 & 0.525 to 0.787
\end{tabular}

Minor CM

One or more

$0.01 \quad 0.598$

0.549 to 0.648

$\mathrm{CM}$, congenital malformation; ROC, receiver operating characteristic

\section{Discussion}

In these infants of mothers with GDM, severity of GDM (early diagnosis and higher 1-h blood glucose in the diagnostic OGTT) was identified in the backward logistic regression analysis as a predictor of one or more major CM. This is consistent with the well-documented pathogenic role of hyperglycaemia in pregnant women with Type 1 and Type 2 diabetes [1,2], and with some recent studies in GDM $[6,7,8]$. To our surprise, the main predictor of one or more major $\mathrm{CM}$ in the present study was higher pre-pregnancy BMI, a variable that was included as a potential confounder because it has been described as a predictor for major CM in the general population [13]. Its relevance comes from being identified in the forward and backward analyses and because it was the main contributor to the area under the ROC curve.

Pre-pregnancy BMI was also the main predictor of the more prevalent major CM (heart and renal/urinary), as well as a predictor of minor CM, the association being always in the positive direction. Severity of GDM was the only predictor of skeletal major CM and was also a predictor of heart CM and minor CM. However, we found negative associations between $\mathrm{CM}$ and blood glucose values in the diagnostic OGTT: 3-h blood glucose was a negative predictor of minor $\mathrm{CM}$, and 2-h blood glucose was a negative predictor of heart CM (non-significant for individual tertiles). The unexpected direction of these relationships between severity of GDM and CM was clearly balanced in the case of major heart CM by the association with abnormal values in the diagnostic OGTT and with gestational age at diagnosis, and to a lesser extent in the case of minor $\mathrm{CM}$ by the association with 2 -h blood glucose in the diagnostic OGTT.

Although the aforementioned studies of $\mathrm{CM}$ in infants of GDM women $[6,7,8]$ do not describe a role for BMI, this could be due to the fact that BMI was not included in the multivariate analysis $[6,7]$, even when there were significant differences in BMI between groups with and without anomalies [8]. Such a contribution of pre-pregnancy BMI to $\mathrm{CM}$ in GDM was unexpected, but its role in the general obstetric population has been firmly established. Indeed, since its initial description in 1969 [15] an association has been described for several types of defects [13], and most papers are concordant for a positive relationship $[13,16,17,18,19,20]$, with a single paper describing no association [21]. A dose-response relationship has been described for neural tube defects $[13,17,18,19]$ and cardiovascular defects [22], and increased BMI accounts for a significant proportion of malformations in the general population $[18,22]$. As to the potential additive effect of diabetes and obesity, we are aware of only one paper [23] describing a threefold risk of $\mathrm{CM}$ in women with obesity and diabetes (either gestational or pre-gestational), but risk was not increased in women with diabetes or obesity alone.

The positive associations of pre-pregnancy BMI and heart and renal/urinary major CM reported in our study for infants of mothers with GDM have counterparts in the general population [22, 24], but to our knowledge no association between minor $\mathrm{CM}$ and obesity has been described. However, we did not find an association between obesity and $\mathrm{CM}$ affecting the central nervous system, which are more characteristically associated with obesity in the general population $[15,16,17$, $18,19,20]$. We attribute this to a low statistical power to detect this association, due to the low rate of central nervous system $\mathrm{CM}$ in this series. Finally, pre-pregnancy BMI was the main predictor of one or more major CM. This was expected, since pre-pregnancy BMI was the main predictor of individual major $\mathrm{CM}$ with higher prevalence (heart and renal/urinary).

Several mechanisms could underlie the association between pre-pregnant maternal obesity and CM. The first is an increased supply of nutrients to the fetus in obese women. In animal models, an excess of several types of fuels (glucose, ketone bodies, NEFA, amino acids) was embryotoxic [25], probably acting through increased oxidative stress [26]. A second possible mechanism is hyperinsulinaemia itself. Insulin was recognised as a teratogen in chicken embryo in 1945 [27]. In the absence of specific antibodies [28], insulin is generally considered not to cross the human placenta [29] but studies do not adequately address early pregnancy. In mammals, moreover, we know that insulin is present in the maternal reproductive tract [30] and that receptors for insulin are present in the embryo as early as the morula stage [30]. It has recently been reported that proinsulin excess in chicken embryos induces teratogenesis by reducing naturally occurring apoptosis [31]. A teratogenic role for insulin in human pregnancy is therefore possible. Interestingly, a recent study identified obesity and hyperinsulinaemia as risk factors for neural tube defects in the general population [32].

We conclude that in infants of mothers with GDM, both BMI and severity of GDM are predictors of CM, with BMI being the main predictor of more prevalent CM. 
Acknowledgements. The Investigation Centre belongs to the Centre Network of the Carlos IIII Health Institute, C03/08, Molecular determinants of Metabolism, Nutrition and Hormonal Biocommunication (2003-2005).

\section{References}

1. Pedersen LM, Tygstrup I, Pedersen J (1964) Congenital malformations in newborn infants of diabetic women. Correlation with maternal diabetic vascular complications. Lancet 13: 1124-1126

2. Towner D, Kjos SL, Leung B et al. (1995) Congenital malformations in pregnancies complicated by NIDDM. Diabetes Care 18: 1446-1151

3. Miller E, Hare JW, Cloherty JP et al. (1981) Elevated maternal hemoglobin A1c in early pregnancy and major congenital anomalies in infants of diabetic mothers. N Engl J Med 304: 1331-1334

4. Ray JG, O'Brien TE, Chan WS (2001) Preconception care and the risk of congenital anomalies in the offspring of women with diabetes mellitus: a meta-analysis. QJM 94: 435-444

5. Martínez-Frías ML, Bermejo E, Rodríguez-Pinilla E, Prieto L, Frías JL (1998) Epidemiological analysis of outcomes of pregnancy in gestational diabetic mothers. Am J Med Genet 78: 140-145

6. Sheffield JS, Butler-Koster EL, Casey BM, McIntire DD, Leveno KJ (2002) Maternal diabetes mellitus and infant malformations. Obstet Gynecol 100: 925-930

7. Schaefer UM, Songster G, Xiang A, Berkowitz K, Buchanan TA, Kjos SL (1997) Congenital malformations in offspring of women with hyperglycemia first detected during pregnancy. Am J Obstet Gynecol 177: 1165-1171

8. Schaefer-Graf UM, Buchanan TA, Xiang A, Songster G, Montoro M, Kjos SL (2000) Patterns of congenital anomalies and relationship to initial maternal fasting glucose levels in pregnancies complicated by type 2 and gestational diabetes. Am J Obstet Gynecol 182: 313-320

9. Metzger BE (1991) Summary and recommendations of the Third International Workshop-Conference on Gestational Diabetes Mellitus. Diabetes 40 [Suppl 2]: 197-201

10. Harris MI, Hadden WC, Knowler WC, Bennett PH (1985) International criteria for the diagnosis of diabetes and impaired glucose tolerance. Diabetes Care 8: 562-567

11. Alberti KG, Zimmet PZ (1998) Definition, diagnosis and classification of diabetes mellitus and its complications. Part 1: diagnosis and classification of diabetes mellitus provisional report of a WHO consultation. Diabet Med 15: 539-553

12. Hollier LM, Leveno KJ, Kelly MA, McIntire DD, Cunningham FG (2000) Maternal age and malformations in singleton births. Obstet Gynecol 96: 701-706

13. Waller DK, Mills JL, Simpson JL et al. (1994) Are obese women at higher risk for producing malformed offspring? Am J Obstet Gynecol 170: 541-548

14. Seidman DS, Ever-Hadani P, Gale R (1990) Effect of maternal smoking and age on congenital anomalies. Obstet Gynecol 76: 1046-1050
15. Richards ID (1969) Congenital malformations and environmental influences in pregnancy. Br J Prev Soc Med 23: 218-225

16. Naeye RL (1990) Maternal body weight and pregnancy outcome. Am J Clin Nutr 52: 273-279

17. Shaw GM, Velie EM, Schaffer D (1996) Risk of neural tube defect-affected pregnancies among obese women. JAMA 275: 1093-1096

18. Watkins ML, Scanlon KS, Mulinare J, Khoury MJ (1996) Is maternal obesity a risk factor for anencephaly and spina bifida? Epidemiology 7: 507-512

19. Kallen K (1998) Maternal smoking, body mass index, and neural tube defects. Am J Epidemiol 147: 1103-1111

20. Shaw GM, Nelson V, Moore CA (2002) Prepregnancy body mass index and the risk of multiple congenital anomalies. Am J Med Genet 107: 253-255

21. Haddow JE, Palomaki GE (1995) Is maternal obesity a risk factor for open neural tube defects? Am J Obstet Gynecol 172: $245-247$

22. Watkins ML, Botto LD (2001) Maternal prepregnancy weight and congenital heart defects in the offspring. Epidemiology 11: 439-446

23. Moore LL, Singer MR, Bradlee ML, Rothman KJ, Milunsky A (2000) A prospective study of the risk of congenital defects associated with maternal obesity and diabetes mellitus. Epidemiology 11: 689-694

24. Queisser-Luft A, Kieninger Baum D, Menger H, Stolz G, Schlaefer K, Merz E (1998). Does maternal obesity increase the risk of fetal abnormalities? Analysis of 20,248 newborn infants of the Mainz Birth Register for detecting congenital abnormalities (in German). Ultraschall Med 19: 40-44

25. Eriksson UJ (1995) The pathogenesis of congenital malformations in diabetic pregnancy. Diabetes Metab Rev 11: 63-82

26. Wentzel P, Thunberg L, Eriksson UJ (1997) Teratogenic effect of diabetic serum is prevented by supplementation of superoxide dismutase and $\mathrm{N}$-acetylcysteine in rat embryo culture. Diabetologia 40: 7-14

27. Landauer W (1945) Rumplessness of chicken embryos produced by the injection of insulin and other chemicals. J Exp Zool 98: 65-77

28. Bauman WA, Yalow RS (1981) Transplacental passage of insulin complexes to antibody. Proc Natl Acad Sci USA 78: 4588-4590

29. Buse MG, Roberts WJ, Buse J (1962) The role of human placenta in the transfer and metabolism of insulin. J Clin Invest 41: 29-41

30. Heyner S, Farber M, Rosenblum IY (1990) The insulin family of peptides in early mammalian development. In: Nilsen-Hamilton M (ed) Current topics in developmental biology, vol 24. Academic Press, New York, pp 137-159

31. Hernández-Sánchez C, Mansilla A, Rosa EJ de la, Pollerberg GE, Martínez-Salas E, Pablo F de (2003) Upstream AUGs in embryonic proinsulin mRNA control its low translation level. EMBO J 22: 5582-5592

32. Hendricks KA, Nuno OM, Suarez L, Larsen R (2001) Effects of hyperinsulinemia and obesity on risk of neural tube defects among Mexican Americans. Epidemiology 12: 630-635 